\title{
Coexistence D’un Lymphome Lymphocytique Et D'une Leucémie Myéloïde Chronique: A Propos De Deux Observations Au Togo
}

\author{
Padaro Essohana, \\ Magnang Hèzouwè, \\ Layibo Yao, \\ Kueviakoe Irénée MD,
}

Service d’hématologieclinique, CHU Campus de Lomé, Faculté des Sciences de la Santé/ Université de Lomé, Togo

\section{Mawussi Koffi,}

Service d'hématologie, CHU Kara, Faculté des Sciences de la Santé /Université de Kara, Togo

Vovor Ahoefa,

Service d'hématologie, CHU Sylvanus Olympio, Faculté des Sciences de la Santé /Université de Lomé, Togo

\section{Doi:10.19044/esj.2021.v17n40p228}

Submitted: 14 August 2021

Accepted: 08 September 2021

Published: 30 November 2021
Copyright 2021 Author(s)

Under Creative Commons BY-NC-ND 4.0 OPEN ACCESS

Cite As:

Essohana P., Hèzouwè M., Yao L., Irénée K., \& Koffi M., \& Ahoefa V.,(2021). Coexistence D’un Lymphome Lymphocytique Et D’une Leucémie Myéloïde Chronique: A Propos De Deux Observations Au Togo. European Scientific Journal, ESJ, 17(40), 228.

https://doi.org/10.19044/esj.2021.v17n40p228

\section{Résumé}

Les auteurs rapportent deux cas de lymphomes lymphocytiques associés à une leucémie myéloïde chronique (LMC) au Togo. La première association est une coexistence simultanée d'une LMC avec présence du transcrit de fusion bcr-abl chez une patiente de 32 ans, au stade myélocytaire chronique, avec une polyadénopathie ayant nécessité une biopsie ganglionnaire qui a conclu à un lymphome lymphocytique. Le deuxième cas est une succession à 6 ans 5 mois d'intervalle, chez un patient de 30 ans, d'une LMC avec présence du bcr-abl et d'un chromosome Philadelphie et d'un lymphome lymphocytique. Ces deux cas sont rapprochés des associations d'hémopathies myéloïdes et lymphoïdes, aiguës ou chroniques. Différentes hypothèses pathogéniques sont passées en revue : responsabilité de la 
chimiothérapie mais le diagnostic est parfois simultané ; implication des oncogènes cellulaires; plus vraisemblablement anomalie clonale de la cellule souche hématopoiétique s'exprimant de façon séquentielle ou simultanée sur les deux lignées filles. A notre connaissance, il s'agit des premières descriptions en Afrique subsaharienne.

Mots clés: Lymphome Lymphocytique, Leucémie Myéloïde Chronique, Lomé (Togo)

\title{
Coexistence of Lymphocytic Lymphoma and Chronic Myeloid Leukemia: A Case of Two Observations in Togo
}

\author{
Padaro Essohana, \\ Magnang Hèzouwè, \\ Layibo Yao, \\ Kueviakoe Irénée MD,
}

Service d'hématologieclinique, CHU Campus de Lomé, Faculté des Sciences de la Santé/ Université de Lomé, Togo

\section{Mawussi Koffi,}

Service d’hématologie, CHU Kara, Faculté des Sciences de la Santé

/Université de Kara, Togo

Vovor Ahoefa,

Service d’hématologie, CHU Sylvanus Olympio, Faculté des Sciences de la Santé /Université de Lomé, Togo

\begin{abstract}
This paper reported two cases of lymphocytic lymphoma associated with chronic myeloid leukemia (CML) in Togo. The first association is a simultaneous coexistence of a CML with the presence of the transcript bcrabl, in a 32-year-old woman, at chronic myelocytic stage with polyadenopathy required and lymph node biopsy concluded for lymphocytic lymphoma. The second is the case of a 30-year-old man who developed a lymphocytic lymphoma after 6years and 5 months of following CML. These two cases are comparable to other associations of acute or chronic myeloid and lymphoid blood malignancies that was previously published. Several pathogenic hypothesis were reviewed which takes responsibility for chemotherapy. Nevertheless, the diagnosis is sometimes simultaneous with the involvement of cellular oncogenes. This is a more likely clonal abnormality of the stem cell sequentially or concomitantly expressed in the cells on both lineages. To the
\end{abstract}


best of our knowledge, these are the first descriptions in South-Saharan Africa

Keywords: Lymphocytic Lymphoma, Chronic Myeloid Leukemia, Lome (Togo)

\section{Introduction}

La leucémie myéloïde chronique (LMC) est un syndrome myéloprolifératif caractérisé par la prolifération des cellules de la lignée granuleuse due à une anomalie chromosomique, le chromosome Philadelphie (Ph1) qui engendre un gène hybride bcr-abl, responsable d'une protéine à forte activité tyrosine kinase (Druker et al., 2001). Les lymphomes non hodgkiniens (LNH) désignent des proliférations clonales de cellules des lignées lymphocytaires $\mathrm{B}$ ou $\mathrm{T}$ à leurs différents stades de différenciation et d'activation. Ils sont responsables du développement de tumeurs au niveau des organes lymphoïdes, notamment ganglionnaires, mais aussi de territoires non lymphoïdes (Cuenca et al., 2009).

La coexistence d'une double prolifération maligne, myéloïde et lymphoïde et particulièrement lymphomateuse, est rare bien que décrite depuis quelques décennies (Martoïa et al., 1987; Doumouchel et al., 1983; Monoharan et al., 1981) et depuis quelques années plusieurs publications décrivent des hémopathies lymphoïdes diversement associées aux hémopathies myéloïdes surtout la LMC (Amy et al., 2018; Xiaorui Fu et al., 2018; Paolo et al., 2018; Smeeta Gajendra et al., 2016). Cette coexistence qu'elle soit spontanée ou non, simultanée ou séquentielle, elle évoque davantage une atteinte de la cellule souche hématopoïétique, se démasquant au niveau de deux lignées filles, qu'une banale coïncidence. A notre connaissance, cette association est très rare en Afrique et au Togo aucun cas n’a été rapporté jusqu'à ce jour. Nous rapportons les deux premiers cas de lymphomes lymphocytiques, l'un diagnostiqué concomitamment avec la LMC et l'autre survenue au cours d'une LMC traitée depuis 6ans 5mois par imatinib.

\section{Observations \\ Patiente 1}

Madame H.K., 32 ans, asthmatique, a été référée de l'hôpital d'Afagnan le 14 janvier 2009 pour prise en charge d'une hyperleucocytose. Le début remonterait à 3 mois plus tôt par la survenue d'une masse à l'hypochondre gauche dans un contexte de sueurs nocturnes, d'amaigrissement et d'aménorrhée non gravidique. Deux mois plus tard, sont apparues des adénopathies cervicales et inguinales. Elle a consulté l'hôpital d'Afagnan d'où elle fut référée devant l'hyperleucocytose à $149.000 / \mathrm{mm}^{3}$. 
L'examen à l'entrée a noté : Poids : 61kg, un ECOG à 2, l'abdomen était ballonné avec une splénomégalie type $\mathrm{V}$ de Hackett, des adénopathies multiples, cervicales gauches dont la plus grosse mesurait $4 \mathrm{~cm}$ et inguinales gauches dont la plus grosse mesurait $5 \mathrm{~cm}$.

L'hémogramme a montré une hyperleucocytose à $174000 / \mathrm{mm}^{3}$ avec une myélémie polymorphe et importante avec myéloblastes à $2 \%$ et promyélocytes à $5 \%$, une anémie à $9,6 \mathrm{~g} / \mathrm{dl}$ non régénérative (réticulocytes : $23000 / \mathrm{mm}^{3}$ ) et une hyperplaquettose à $635000 / \mathrm{mm}^{3}$. Un myélogramme réalisé le même jour a confirmé l'hyperplasie granuleuse médullaire à $88 \%$ avec myéloblastes à $2,5 \%$ et promyélocytes à $6,5 \%$. Une leucémie myéloïde chronique au stade myélocytaire chronique a été fortement suspectée et confirmée 6 semaines plus tard par la présence du transcrit de fusion bcr-abl de type b2-a2 réalisé à l’hôpital Henri Mondor à Créteil (France). Devant cette polyadénopathie importante à ce stade de la LMC, nous avons réalisé dans la foulée une biopsie ganglionnaire qui a retrouvé une prolifération de petits lymphocytes matures avec une chromatine dense, d'architecture diffuse pouvant être compatible avec un lymphome lymphocytique. L’immunomarquage n'a pu être réalisé (plateau technique limité). Les autres bilans biologiques ont montré une augmentation des LDH à 1443 UI/l, une augmentation des PAL à 802UI/L, une augmentation de gamma GT, l'acide urique était normal à 38mg/l, la sérologie VIH négative, la VS était normale à $16 \mathrm{~mm}$, l'électrophorèse des protéines sériques était normale, le bilan hépatique et rénal normal. L'échographie abdominale objective une hépatosplénomégalie homogène avec signe d'hypertension portale, une souffrance rénale bilatérale type I et des adénopathies profondes. La TDM, l'immunophénotypage et le caryotype n’ont pu être réalisés faute de moyens financiers.

La patiente a été mise d'emblée sous hydroxyurée à 30mg/Kg/jour associé à l'allopurinol 100mg/ jour et une hyperhydratation. Ce traitement a été remplacé par l'imatinib mésylate $400 \mathrm{mg} /$ jour au 15è jours après la confirmation diagnostique par la présence du transcrit de fusion bcr-abl et la patiente incluse dans le programme GIPAP (Glivec International Patients Assistance Programm) grâce auquel les patients bénéficient gratuitement de Glivec* au Togo. Pour son lymphome, une chimiothérapie de type mini CHOP (Cyclophosphamide, Doxorubicine, Vincristine, Prednisolone) a été préconisée mais l'ordonnance des antimitotiques n'a pu être honorée par manque de moyens financiers.

Sous hydroxyurée puis imatinib, on a noté un début de rémission clinique et hématologique puisque à J21, la splénomégalie était de type III, les globules blancs étaient à $37000 / \mathrm{mm}^{3}$. Malheureusement la patiente a été perdue de vue et de contact et est revenue le 23 mars 2010 (soit 5 mois 9 jours plus tard) dans un tableau d'altération de l'état général, une augmentation du 
syndrome tumoral avec une splénomégalie de type IV, des œdèmes des membres inférieurs, et surtout une dyspnée avec douleurs thoraciques dont l'exploration a noté une péricardite avec début de tamponnade. Elle est décédée le 29 mars 2010.

\section{Patient 2}

Monsieur M.K., 30 ans, a été référé de l'hôpital secondaire de Bè le 08 août 2012 pour hyperleucocytose à $234900 / \mathrm{mm}^{3}$. Un mois plus tôt, il a présenté des douleurs articulaires, une sensation de lourdeur de l'hypochondre gauche et une plénitude gastrique. Il a consulté à l'hôpital secondaire de Bè puis référé. L'examen à l'entré a noté : Poids : $61 \mathrm{Kg}$, un très bon état général (ECOG : 0) une splénomégalie type $\mathrm{V}$ de Hackett sans hépatomégalie, toutes les aires ganglionnaires étaient libres.

L’hémogramme a objectivé une hyperleucocytose à 396000/ $\mathrm{mm}^{3}$ avec une myélémie polymorphe et importante faite de myéloblastes à $2 \%$ et promyélocytes à $8 \%$, une anémie à $10,8 \mathrm{~g} / \mathrm{dl}$ arégénérative (réticulocytes : $37000 / \mathrm{mm}^{3}$ ) et une hyperplaquettose à $568000 / \mathrm{mm}^{3}$. Un myélogramme a confirmé l'hyperplasie granuleuse médullaire à $91,5 \%$ avec myéloblastes à $3,5 \%$ et promyélocytes à $5,5 \%$. Une leucémie myéloïde chronique au stade myélocytaire chronique a été fortement suspectée et confirmée 15 jours plus tard par la présence du bcr-abl de type b3-a2 réalisé à l’hôpital Henri Mondor à Créteil (France). Les autres bilans biologiques ont montré une augmentation des LDH à 2829UI/l, une hyperuricémie à 94mg/l, le taux des PAL était normal à 356 UI/L, la sérologie VIH négative, présence de l'antigène HbS, la VS était normale à $16 \mathrm{~mm}$, l'électrophorèse des protéines sériques était normale, le bilan hépatique et rénal normal.

D’emblée le patient a été inclus au programme GIPAP puis mis sous imatinib mésylate $400 \mathrm{mg} / \mathrm{j}$ our, dose qui a été réduite à $300 \mathrm{mg}$ puis à $200 \mathrm{mg}$ en raison des neutropénies profondes. Sous ce traitement on a noté une rémission clinique puis hématologique puisque au contrôle du 12 Juin 2014 (soit 22 mois après) il n'avait pas de splénomégalie et l'hémogramme montrait 4600 leucocytes $/ \mathrm{mm}^{3}$ dont 2070 polynucléaires neutrophiles $/ \mathrm{mm}^{3}$.

A partir du 18 septembre 2014 (soit 2ans 1mois après) on a constaté une augmentation progressive de la leucocytose atteignant parfois $21000 / \mathrm{mm}^{3}$ (sans signes d'accélération ni d'acutisation) pour laquelle la dose d'imatinib a été réajustée à $300 \mathrm{mg}, 400 \mathrm{mg}$ puis à $600 \mathrm{mg} /$ jour sans succès. Dans l'hypothèse de l'apparition d'une nouvelle mutation résistante à l'imatinib, un caryotype médullaire a été fait le 29 mai 2016 au laboratoire CERBA en France et a confirmé la présence du chromosome Philadelphie sans aucune autre anomalie surajoutée.

Dans un premier temps le patient a été mis sous imatinib associé à l'hydroxyurée puis à partir de 23 octobre 2018 sous imatinib seul. 
Parallèlement à partir du 20 février 2018 (5ans 7mois après) on a noté des adénopathies cervicales droites multiples infracentimétriques dont la taille a augmenté spontanément en décembre 2018 pour atteindre 2,5cm alors que la rémission hématologique est maintenue avec l'hémogramme du 14 janvier 2019 qui montrait des leucocytes à $4200 / \mathrm{mm}^{3}$ avec des polynucléaires neutrophiles à $2100 / \mathrm{mm}^{3}$ et des lymphocytes à $1806 / \mathrm{mm}^{3}$. Une biopsie ganglionnaire a alors été faite le 15 janvier 2019 (6ans 5mois). L'examen anatomopathologique retrouve une prolifération de petits lymphocytes matures, d'architecture diffuse avec des zones nodulaires de cellules plus nucléolées pouvant être compatible avec un lymphome lymphocytique. L’immunomarquage n'a pu être réalisé (plateau technique limité). L'échographie abdominale et la radiographie du thorax n'ont pas retrouvé d'adénopathies profondes. Le caryotype et le scanner n’ont pu être réalisés pour manque de moyens financiers. Il a un bon état général (ECOG : 1) avec des taux normaux de LDH à 194UI/L et des Béta 2 microglobulines à 1,9mg/l. Le reste du bilan biologique est relativement normal. Une abstention thérapeutique a été préconisée avec une surveillance très rapprochée.

\section{Discussion}

Le diagnostic des hémopathies malignes, à la fois myéloïde et lymphoïde chez un même patient, qu'il soit simultané ou séquentiel, est extrêmement rare, avec une incidence globale inférieure à 1\% (Hauck et al., 2013). La majorité des cas (66\%) ont des présentations séquentielles alors que seulement 34\% sont présents simultanément (Laurenti et al., 2011).

Chez notre première patiente, le diagnostic de la LMC et du LNH a été simultané alors que chez le 2è patient les deux affections se sont succédé à 6 ans 5 mois d'intervalle. Une LMC au stade myélocytaire chronique a été suspectée par la cytologie sanguine et médullaire et confirmée par la présence du transcrit bcr-abl chez la première patiente et chez le 2è patient par la présence du chromosome Philadelphie. Chez nos deux patients, un LNH de faible malignité histologique puisque d'architecture lymphocytique à petites cellules donc excluant l'hypothèse d'une crise blastique sur un mode lymphoïde. Des LNH de faible malignité survenue simultanément ou au décours de l'évolution d'une LMC ont été déjà rapportées par trois auteurs (Martoïa et al., 1987; Amy et al., 2018; Fujiwara et al., 2018). En Afrique sud saharienne, il s'agit des toutes premières observations à notre connaissance. Des adénopathies sont inhabituelles, présentes seulement dans 7\% des 161 LMC suivies par Paolino et al. (1980); leur signification n'est pas univoque : ces adénopathies, de petites à toute petites, plus ou moins généralisées, elles relèvent d'une simple métaplasie myéloïde (3\% des LMC); plus volumineuses voire pseudo-sarcomateuses dans 5\% des LMC en transformation aiguë selon Rosenthal et al. (1977), de caractère 
myéloblastique plus souvent que lymphoblastique, elles accompagnent la crise blastique médullo-sanguine, parfois la précèdent de 2 à 26 mois ou encore la suivent de 3 à 4 mois. Une duplication du Ph1 est fréquemment observée (Martoïa et al., 1987). Autrefois exceptionnel, le tableau clinique et l'histologie ganglionnaire correspondant à un LNH authentique agressif au cours d'une LMC, une acutisation sur un mode lymphoblastique étant formellement écarté, cette situation a été rapportée plus tard par plusieurs auteurs (Martoïa et al., 1987). Nos patients avaient des adénopathies qui rentraient dans le cadre d'un lymphome indolent et n'étaient pas au stade d'acutisation.

Au cours de la LMC, les proliférations clonales de cellules des lignées lymphocytaires à leurs différents stades de différenciation surviennent généralement au cours de la phase accélérée ou acutisée et représentent 15\% des LMC nouvellement diagnostiquées et les territoires les plus fréquemment impliquées dans ces proliférations sont les ganglions lymphatiques, les os, la peau, les tissus mous et le système nerveux central (Amy et al., 2018).

La première association d'un LNH et d'une LMC a été faite pour la première fois par Hanns et al. (1934) et Martoïa et al. (1987) qui ont colligé 24 cas certains sans prédominance d'un type histologique et la chronologie de survenue des deux pathologies montrait que dans 14 cas, la LMC précède le LNH de 6 mois à 6 ans (soit 58,33\% des cas), toujours traitée ; l'inverse ne survient que 2 fois dans un délai de 2 à 3 ans. Le diagnostic était spontané 8 fois. La LMC est toujours en phase chronique lorsque le lymphome apparait ; une transformation aiguë ne survient que 6 fois sur 24 en 12 mois; et aucun parallélisme évolutif ne peut être retenu entre la LMC et le lymphome qui, généralement, domine le pronostic à court ou à moyen terme. Dans notre étude, le diagnostic des deux pathologies a été simultané dans 50\% des cas et survenue plus tard dans $50 \%$ de cas après un traitement de 6 ans 5 mois. Nos résultats se rapprochent donc de ceux de la littérature (Martoïa et al., 1987).

Par contre dans son étude Inverardi et al. (1990) avaient trouvé que la moitié des patients étaient en phase chronique lors de la prolifération lymphoïde et l'autre moitié était en phase accélérée ou acutisée. Les patients qui étaient en phase accélérée ont progressé vers la phase aiguë après 4 mois, impliquant que la prolifération lymphoïde peut annoncer une phase imminente d'acutisation même si la transformation blastique était absente initialement (Inverardi et al., 1990). Une surveillance s'avère donc nécessaire pour ne pas méconnaitre une acutisation chez notre 2è patient. Chez la première patiente le tableau clinique présenté peu avant le décès peut être lié à l'évolution naturelle du LNH mais aussi à une acutisation. Malheureusement nous n’avons pu évaluer aucune des deux éventualités avant son décès.

Toutes ces premières études ont évalué les patients avant l'avènement des inhibiteurs de la tyrosine kinase (ITK) qui ont révolutionné le traitement et le 
pronostic de la LMC. Néanmoins même sous ITK, il est indispensable de maintenir le suivi pour ne pas méconnaitre une prolifération lymphoïde annonciatrice d'une éventuelle transformation blastique. Dans notre étude, les deux patients ont été mis sous ITK (imatinib). Le deuxième patient, après 6 mois de traitement a présenté des adénopathies alors qu'il était en rémission hématologique et il n'existait pas de signes cytologiques en faveur d'une accélération ou d'une transformation blastique. C'est ce qui nous a poussé à réaliser cette biopsie ganglionnaire qui a retrouvé ce LNH indolent. La même situation a été rapportée par Amy et al. (2018).

La coexistence d'une LMC et d'un LNH est à rapprocher des autres combinaisons, spontanées, de syndromes myéloprolifératifs et lymphoprolifératifs généralement de type $B$ :

- La plus récente est la coexistence d'une leucémie myélomonocytaire chronique (LMMC) et l'un lymphome nodulaire de la zone marginale (Paolo et al., 2018);

- La LMC associée à une maladie de Hodgkin (Ritch et al., 1983), une leucémie lymphoïde chronique (Vilpo et al., 1980), un myélome multiple (Doumouchel et al., 1983);

- Une maladie de Vaquez associée à un LNH, un myélome multiple (Heinle et al., 1996), une LLC (Monoharan et al., 1981);

- Une splénomégalie myéloïde associée à une maladie de Waldenström (Broussier et al., 1976), à un LNH (Epstein et al., 1985).

Leur signification mérite d'être discutée, même si aucune conclusion formelle ne peut être retenue. Une coexistence fortuite paraît hautement improbable en raison de la rareté relative de chacune des deux pathologies (Monoharan et al., 1981). Les oncogènes cellulaires localisés sur les segments chromosomiques engagés dans la transformation $t(9 ; 22)$ (q34; q11), retrouvée chez notre deuxième patient, peuvent être impliqués. Ainsi l'expression de l'oncogène cabl situé en position q34 sur le chromosome 9, se trouve amplifiée par la translocation ; il a été isolé d'un rétrovirus, le virus d'Abelson, inducteur de lymphocytes pré-B chez la souris (Martoïa et al., 1987).

Le traitement de l'affection première pourrait jouer un rôle facilitant, par le biais de l'immunodépression induite : on connait la fréquence des $\mathrm{LNH}$ et des cancers chez les sujets atteints de déficits immunitaires congénitaux ou acquis (surtout depuis la survenue de la pandémie du VIH), iatrogènes ou secondaires à un syndrome lymphoprolifératif (Epstein et al., 1985). Certes à chaque fois que la LMC a précédé le lymphome (ou lui a succédé), on retrouvait une irradiation et/ou un traitement par alkylants dont le rôle mutagène et oncogène n'est plus à discuter ; en déduire que le lymphome trouve son origine dans le traitement de la LMC (ou l'inverse), donc ne retenir qu'une relation indiscrète, n'expliquerait pas le petit nombre de cas décrits, les délais de survenue parfois très courts (de quelques mois seulement) et surtout 
les observations où le diagnostic est simultané (Doumouchel et al., 1983; Monoharan et al., 1981) comme pour notre première patiente.

C'est pourquoi l'hypothèse d'une maladie de la cellule souche hématopoïétique, extériorisée à deux niveaux et sur deux lignées filles, paraît comme la plus séduisante et la plus conforme aux conceptions actuelles qui font de la LMC une maladie clonale d'une cellule totipotente, précurseur commun des lignées lymphoïdes et myéloïdes (Monoharan et al., 1987) comme viennent l'appuyer la présence du $\mathrm{Ph} 1$ dans les lymphocytes $\mathrm{B}$ matures et dans les transformations aiguës sur un mode lymphoblastique.

Depuis quelques années, les études cytogénétiques et moléculaires sur les oncogènes cellulaires ont permis d'établir des liens de parenté entre les processus prolifératifs apparemment distincts. En effet les mutations génétiques des oncogènes tels que ceux de la famille du RAS, ou des gènes suppresseurs de tumeurs, tels que p53, ont été retrouvés chez les patients atteints de LMC et de LNH (Hirsch-Ginsberg et al., 1990; Nakatsuka et al., 2002) et théoriquement prédisposés à plusieurs tumeurs malignes. Dans une étude récente, les données de l'étude de contrôle IV randomisée, étude IV de la LMC, ont été analysées pour évaluer l'impact de l'utilisation à long terme des ITK dans le développement de tumeurs malignes secondaires. Les patients LMC sous ITK avaient une incidence standard significativement plus élevée (ratio de 3,33 pour les hommes et de 4,29 pour les femmes) de développer un LNH par rapport à une population allemande. Le délai médian entre le diagnostic de LMC et le diagnostic d'une autre tumeur maligne était de 2,4 ans. En effet les mécanismes de réparation de l'ADN par les ITK pourraient être un potentiel mécanisme de cette découverte basé sur des études précliniques (Amy et al., 2018). Nos deux patients ont bénéficié d'un traitement par ITK qui a permis d'obtenir une bonne rémission clinique et hématologique et le 2è patient n’a développé son lymphome qu'après un long délai de 6 ans 5 mois.

Les onco-hématologistes et pathologistes doivent être conscients de la possibilité de tumeurs malignes hématologiques concomitantes. Une adénopathie chez un patient atteint de LMC peut représenter une transformation blastique, mais une hémopathie lymphoïde distincte peut également être possible. Lorsque cela est suspecté à l'examen clinique, il est indispensable d'effectuer une évaluation plus poussée en utilisant de nouvelles techniques notamment l'immunohistochimie, la cytométrie en flux, la PCR qui permettent un diagnostic concluant et précis. Comme le traitement de chacune des hémopathies malignes diffère souvent, cette distinction est importante pour permettre aux cliniciens de mieux poser de bonnes indications thérapeutiques. 


\section{Conclusion}

La séquence LMC - LNH mérite encore d'être rapportée au Togo et en Afrique subsaharienne, non par esprit de collection, mais par intérêt dogmatique et doctrinal. Une banale coïncidence est à écarter, en raison des multiples descriptions de syndromes myéloprolifératifs et lymphoprolifératifs associés. La LMC a valeur d'exemplarité car elle apporte des arguments solides pour une maladie clonale originelle. Des études ultérieures pour évaluer la pathogenèse de la double existence des proliférations malignes, myéloïdes et lymphoïdes, sont nécessaires pour mieux comprendre et gérer les patients.

\section{References:}

1. Amy G. Starr, Sushma R. Jonna, Joeffrey J. Chahine, Bhaskar V. Kallakury, \& Chaitra S. Ujjani (2018). Concurrent Diagnosis of Chronic Myeloid Leukemia and Follicular Lymphoma: An Unreported Presentation. Hindawi. Case Reports in Hematology Volume, Article ID 7493601, 4 pages https://doi.org/10.1155/2018/7493601

2. Broussier, J., Dao, C., \& Zittoun, R. (1976). Macroglobulinémie de Waldenström avec splénomégalie myéloïde. Ann Med Interne; 127 : 483-90.

3. Cuenca, X., Xhaard, A., \& Mounier. N. (2009). Facteurs pronostiques dans les lymphomes non hodgkiniens et les lymphomes de hodgkin. Bulletin du cancer; 96 : 461- 473.

4. Doumouchel, P., Casassus, Ph., Giocomini, T., \& Lortholary, P. (1983). Coexistence d'un myélome et d'une leucémie myéloide chronique. Sem Hôp (Paris); 59 : 2191 - 3

5. Druker, BJ., Talpaz, M., Resta, DJ., Peng, B., Buchdunger, E., Ford, JM et al. (2001). Efficacy and safety of a specific inhibitor of the BCRABL tyrosine kinase in chronic myeloid leukemia. N Engl J Med; 344: 1031-7

6. Epstein, JR., Joshua, DE., Kronenberg, H. (1985). idiopathic myelofibrosis complicated by lymphoma. Report of two cases. Acta Haematol ; $73: 40-4$.

7. Fujiwara, S. I., Shirato, Y., Ikeda, T. et al. (2018). Successful treatment of follicular lymphoma with second-generation tyrosine kinase inhibitors administered for coexisting chronic myeloid leukemia," International Journal of Hematology ; 107(6) :712-715.

8. Hanns, A., Israel, L., Sacrez, R. (1934). Sarcome lymphoblastique avec image sanguine de leucémie myéloïde chronique. Strasbourg Med; 94 : 42 - 3. 
9. Hauck, G., Jonigk, D., Kreipe, H. \& Hussein, K. (2013). "Simultaneous and sequential concurrent myeloproliferative and lymphoproliferative neoplasms," Acta Haematologica; 129 (3) : 187196.

10. Heinle, EW., Sarasti, HO., Garcia, D., Kenny, JJ., \& Werterman, MP. (1996). Polycthemia vera associated with lymphomatous disease and myeloma. Arch Intern Med; 118 : 351-55.

11. Hirsch-Ginsberg, C., LeMaistre, A. C., Kantarjian, H. et al. (1990). RAS mutations are rare events in Philadelphia chromosomenegative/ bcr gene rearrangement-negative chronic myelogenous leukemia, but are prevalent in chronic myelomonocytic leukemia. Blood $1990 ; 76$ : $1214-19$.

12. Inverardi, D., Lazzarino, M., Morra, E. et al. (1990). Extramedullary disease in $\mathrm{Ph}$ '-positive chronic myelogenous leukemia: frequency, clinical features and prognostic significance. Haematologica ; 75 : 146-148.

13. Laurenti, L., Tarnani, M., Nichele, I. et al. (2011). The coexistence of chronic lymphocytic leukemia and myeloproliperative neoplasms: a retrospective multicentric GIMEMA experience,”American Journal of Hematology; 86(1) : 1007-1012.

14. Martoïa, R., Lamy, T., Delmaire, P., Algayres, J-P., Rougier, Y., \& Laurens, A. (1987). Survenue d'un lymphome non hodgkinien au cours d'une leucémie myéloïde chronique. Rev Méd Interne; 8 : 471474.

15. Monoharan, A., Catovsky, D., Clein, P et al. (1981). Simultanous or spontaneous occurrence of lympho and myeloproliferativ disorders : a report of four cases. Br J Haemat; 48: 111-6.

16. Nakatsuka, S., Hongyo, T., Syaifudin, M., Nomura, T., Shingu, N., \& Aozasa, K. (2002). Mutations of p53, c-kit, K-ras, and beta-catenin gene in non-Hodgkin's lymphoma of adrenal gland. Japanese Journal of Cancer Research; 93(3): 267-274.

17. Paolo K. Soriano, Taylor Stone, Junaid Baqai, \& Sherjeel Sana (2018). A Case of Synchronous Bone Marrow Chronic Myelomonocytic Leukemia (CMML) and Nodal Marginal Zone Lymphoma (NMZL). American Journal of Case Reports; 19: 1135-1139.

18. Paulino, W., Infelise, V., Degani, G. et al. (1980). Adénopathie nella leucemie mieloide cronica. Min Med; 71 : 2115 - 23.

19. Ritch, PS., Anderson, T., Hanson, GA. et al. (1983). Chronic Granulocytic Leukemia following radiation therapyfor Hodgkin's disease. Cancer; 52 : 462- 4. 
20. Rosenthal, S., Canellos, GP., De Vita, VT Jr., Gralnick, HR. (1977). Characteristics of blast crisis in chronic granulocytic leukemia. Bllod; $49: 705-14$.

21. Smeeta Gajendra, Archana Sharma, Rashi Sharma, Sunil Kumar Gupta, Nitin Sood, \& Ritesh Sachdev (2016). Hodgkin Lymphoma in a Case of Chronic Myeloid Leukemia Treated with Tyrosine Kinase Inhibitors. Case Report. tjpath.01368. doi: 10.5146.

22. Vilpo, JA., Klemi, P., Lassilia, O., \& De la Chapelle, A. (1980). Concomitant presentation of two chronic leukemias : evidence for independant clonal evolution. Am J Hematol; 8 :205 11.

23. Xiaorui Fu, Yufeng Shang, Lei Zhang, Ling Li, Xin Li, Xinhua Wang et al. (2018). Analyses and treatment of simultaneous bi-lineage malignancies of myeloid leukemia and lymphoma: Two case reports and a literature review. Oncology Letters; 16: 6624-6632. 